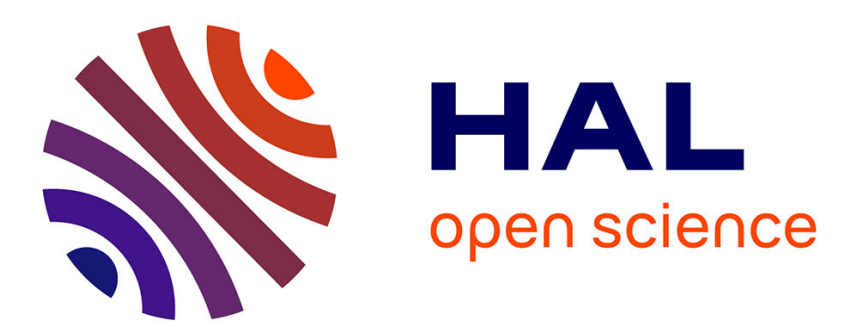

\title{
Sensor placement for field reconstruction in rotating electrical machines
}

\author{
Stéphane Clenet, Thomas Henneron, Julien Korecki
}

\section{To cite this version:}

Stéphane Clenet, Thomas Henneron, Julien Korecki. Sensor placement for field reconstruction in rotating electrical machines. IEEE Transactions on Magnetics, In press, 10.1109/tmag.2021.3072095 . hal-03218208

\section{HAL Id: hal-03218208 \\ https://hal.science/hal-03218208}

Submitted on 5 May 2021

HAL is a multi-disciplinary open access archive for the deposit and dissemination of scientific research documents, whether they are published or not. The documents may come from teaching and research institutions in France or abroad, or from public or private research centers.
L'archive ouverte pluridisciplinaire HAL, est destinée au dépôt et à la diffusion de documents scientifiques de niveau recherche, publiés ou non, émanant des établissements d'enseignement et de recherche français ou étrangers, des laboratoires publics ou privés. 


\title{
Sensor placement for field reconstruction in rotating electrical machines
}

\author{
S. Clénet ${ }^{1}$, T. Henneron ${ }^{2}$ J. Korecki ${ }^{2}$ \\ ${ }^{1}$ Arts et Métiers, Univ. Lille, Centrale Lille, HEI, HESAM Université, EA 2697 - L2EP, F-59000 Lille, France \\ ${ }^{2}$ Univ. Lille, Centrale Lille, Arts et Métiers, HEI, EA 2697 - L2EP, F-59000 Lille, France
}

\begin{abstract}
A method is proposed to place sensors in an electrical machine in order to be able to reconstruct the magnetic field distribution. This method is based on the Empirical Interpolation Method combined with the Maxvol technique. The results applied on a surface mounted permanent magnet machine at no load show that the field distribution can be accurately reconstructed even when the sensor location is imposed in the airgap of the rotating machine.
\end{abstract}

\section{Index Terms - Electrical machines, Field reconstruction, Sensor placement, Finite Element Method}

\section{INTRODUCTION}

$\mathrm{B}$ eing able to reconstruct the magnetic flux density distribution in an electrical machine from several local field measurements would be of great interest enabling to estimate global and local quantities (iron losses, torque...) in real time. With the development of IoT (Internet of Things) combined with the progress in microelectronics, we can expect, in a next future, to equip electrical machines with wireless sensors of magnetic field, temperature... at low cost. The question, which arises, is then: are we able to reconstruct the field distribution in an electrical machine from a limited number of sensors and where shall we locate them?

It seems that this issue of sensor placement has not been addressed in the case of electrical machines especially for internal field reconstruction.

Besides, in numerous research fields, methods have been proposed to find the best position of sensors. Some methods are based on the derivation of the sensor location from Partial Derivative Equations (PDE) model solved analytically [1] or numerically like the (Discrete) Empirical Interpolation Method ((D)EIM) [2,3]. This last method has been applied in the case of nuclear reactor [4]. Moreover, in the field of computational electromagnetics, the (D)EIM has been already applied with success to reconstruct the distribution of the electromagnetic field in the regions where the behavior law is nonlinear, when constructing a reduced order model from a Finite Element (FE) model [5][6]. For all these reasons, the (D)EIM seems to be a good candidate for sensor placement for field reconstruction on the whole electrical machine.

In this paper, a method based on (D)EIM is investigated to place sensors in order to reconstruct the field distribution in a rotating electrical machine. The (D)EIM is coupled with the Maxvol technique to improve its efficiency [7]. The method is tested on a permanent magnet machine.

\section{METHOD FOR SENSOR PLACEMENT}

\section{A. Description of the problem}

Let us consider a FE model of an electrical rotating machine. We denote $n_{e}$ the number of mesh elements, $\theta$ the rotor position and $\mathbf{B}(\theta), 2 \mathrm{n}_{\mathrm{e}} \times 1$, the vector containing the radial and tangential components $\left[b_{i}(\theta)\right]_{1 \leq i \leq 2 n e}$ of the magnetic flux density on each element. Let us introduce the vector $\mathbf{B}^{\operatorname{sens}}(\theta), \mathbf{M} \times 1$, of the magnetic flux density measured by $M$ sensors which represent in fact $M$ entries of $\mathbf{B}(\theta)$. We denote $\varphi, M \times 1$, the vector of the indices of these $\mathrm{M}$ entries. We have then:

$$
\mathbf{B}^{\text {sens }}(\theta)=\left[b^{\text {sens }}(\theta), \ldots, b^{\text {sens }}{ }_{M}(\theta)\right]^{t}=\left[b_{\varphi(1)}(\theta), \ldots, b_{\varphi(M)}(\theta)\right]^{t}
$$

The problem of sensor placement consists then in finding the vector of indices $\varphi$ and the $2 n_{e} \times M$ matrix $\boldsymbol{R}=\left[\mathbf{r}_{\mathrm{i}}\right]_{1 \leq i \leq \mathrm{M}}$, such that the error between $\mathbf{B}(\theta)$ and the reconstructed field

$$
\mathbf{B}^{\text {reconst }}(\theta)=\boldsymbol{R} \mathbf{B}^{\text {sens }}(\theta)
$$

is minimum. Once $\boldsymbol{\varphi}$ and $\boldsymbol{R}$ are determined, the magnetic flux density distribution $\mathbf{B}^{\text {reconst }}(\theta)$ is reconstructed from $\mathbf{B}^{\text {sens }}(\theta)$ gathering $\mathbf{M}$ radial or tangential components of the magnetic flux density of $\mathbf{B}(\theta)$.

The problem of choosing the best vector of indices $\varphi$, which is equivalent to finding the best sensor locations, is combinatorial, which is computationally unfeasible. In fact, the complexity of this combinatorial optimization problem is proportional to $\left(\begin{array}{c}2 \mathrm{n}_{\mathrm{e}} \\ \mathrm{M}\end{array}\right)$ which represent the number of the Mcombinations of the $2 n_{e}$ entries of $\mathbf{B}(\theta)$. For example, let us consider a mesh with $n_{e}=30000$ elements and a sensor number $\mathrm{M}=10$, the $\mathrm{M}$-combination number of sensors is equal to 1.62 $10^{38}$. As an alternative, we have applied the (D)EIM [1][2] combined with the Maxvol algorithm [3]. It enables to obtain quickly a suboptimal sensor location $\varphi$ as well as the reconstruction matrix $\boldsymbol{R}$. The complexity of this algorithm is proportional to the number of sensors $M$. In the following, we present the (D)EIM and then the Maxvol algorithm.

\section{B. (D)EIM}

The aim of the (D)EIM is to approximate the $2 \mathrm{n}_{\mathrm{e}} \times 1$ vector $\mathbf{B}(\theta)$ by a $2 n_{e} \times 1$ vector $\mathbf{B}^{\text {reconst }}(\theta)$ in the form $[2,3]$ :

$$
\mathbf{B}(\theta) \approx \mathbf{B}^{\text {reconst }}(\theta)=\boldsymbol{U} \mathbf{c}(\theta)=\sum_{\mathrm{i}=1}^{\mathrm{M}} \mathrm{c}_{\mathrm{i}}(\theta) \mathbf{U}_{\mathrm{i}}
$$


with $\mathbf{U}_{\mathrm{i}}$ the $\mathrm{i}^{\text {th }}$ column of a $2 \mathrm{n}_{\mathrm{e}} \times \mathrm{M}$ matrix $\boldsymbol{U}$ and $\mathbf{c}(\theta)$ is a vector which entries $\left[c_{1}(\theta), \ldots, c_{M}(\theta)\right]$ being linear combinations of $\mathbf{M}$ "well-chosen entries" of the vector $\mathbf{B}(\theta)$. Then, it is possible to "measure" only $\mathbf{M}$ entries of $\mathbf{B}(\theta)$ to compute $\mathrm{c}_{\mathrm{i}}(\theta)$ and thus, by applying (1), to determine an approximation $\mathbf{B}^{\text {reconst }}(\theta)$ of $\mathbf{B}(\theta)$. In order to determine these $M$ entries, snapshots of $\mathbf{B}(\theta)$ (i.e. solutions of the FE model) are calculated for $\mathrm{N}$ angular positions of the rotor $\left(\theta_{1}, \ldots, \theta_{\mathrm{N}}\right)$. Then, the approximation $\mathbf{B}^{\text {reconst }}(\theta)$ is sought in a subspace $K^{M}$ spanned by an orthogonal basis $\boldsymbol{U}=\left[\mathbf{U}_{1}, \ldots, \mathbf{U}_{\mathrm{M}}\right]$. This basis can be determined by a Gram-Schmidt Process or a Singular Value Decomposition (SVD) of the snapshot matrix $\boldsymbol{B}=\left[\mathbf{B}\left(\theta_{1}\right), \ldots\right.$, $\left.\mathbf{B}\left(\theta_{n}\right)\right]$. In our case, we have considered the $M$ singular vectors $\mathbf{U}_{\mathrm{i}}$ associated to the $\mathrm{M}$ most significant singular values of the SVD of the matrix $\boldsymbol{B}$. Then, by applying a greedy algorithm, the $M$ entries of $\mathbf{B}(\theta)$ are determined and stored in the $M \times 1$ index vector $\varphi$. Only the entries $\left[\mathrm{b}_{\varphi(1)}(\theta), \ldots, \mathrm{b}_{\varphi(\mathrm{M})}(\theta)\right]$ need to be calculated to determine the vector $\mathbf{c}(\theta)$ and so $\mathbf{B}^{\text {reconst }}(\theta)$ from (2). Let $\boldsymbol{P}$ denote the $\mathrm{N} \times \mathrm{M}$ matrix such that the $\mathrm{j}^{\text {th }}$ column is the $\varphi(\mathrm{j})^{\text {th }}$ column $\mathbf{e}_{\varphi(\mathrm{j})}$ of the identity matrix $\boldsymbol{I}_{\mathrm{NxN}}$. All the entries of $\boldsymbol{P}$ are equal to zero except the $M$ entries $p_{\varphi(j), j}(1 \leq j \leq M)$. The vector $\mathbf{c}(\theta)$ is then given by:

$$
\mathbf{c}(\theta)=\left(\boldsymbol{P}^{\mathrm{t}} \boldsymbol{U}\right)^{-1} \boldsymbol{P}^{\mathrm{t}} \mathbf{B}(\theta)
$$

The $\mathbf{M} \times \mathbf{M}$ matrix $\left(\boldsymbol{P}^{\mathrm{t}} \boldsymbol{U}\right)^{-1}$ can be precalculated and the $\mathrm{M}$ entries of the vector $\boldsymbol{P}^{\mathbf{t}} \mathbf{B}(\theta)$ are equal to the $\mathbf{M}$ entries of $\mathbf{B}(\theta)$ gathered in the $M \times 1$ vector $\mathbf{B}^{\operatorname{sens}}(\theta)$ introduced in (1). Then, combining (3) and (4), the expression of the matrix $\boldsymbol{R}$ is given by:

$$
\boldsymbol{R}=\boldsymbol{U}\left(\boldsymbol{P}^{\mathrm{t}} \boldsymbol{U}\right)^{-1}
$$

which enables to reconstruct the field distribution $\mathbf{B}^{\text {reconst }}(\theta)$ by (2).

\section{A. Maxvol technique}

The algorithm Maxvol is an iterative process which aims at finding a $\mathrm{M} \times \mathrm{M}$ submatrix $\boldsymbol{M}^{\text {sub }}$ of the $\mathrm{N} \times \mathrm{M}$ matrix $\boldsymbol{M}(\mathrm{N}>\mathrm{M})$ with an almost maximum volume (the volume of a square matrix $\boldsymbol{M}^{\text {sub }}$ is given by $\operatorname{vol}\left(\boldsymbol{M}^{\text {sub }}\right)=\left|\operatorname{det}\left(\boldsymbol{M}^{\text {sub }}\right)\right|$. The problem of finding the matrix with the maximum volume is combinatorial. The Maxvol technique enables to obtain a suboptimal solution in a reasonable time [9]. The input is the index set $\varphi$ obtained with the (D)EIM describes in the previous section. The output is new index set $\varphi$ from which a new matrix $\boldsymbol{R}$ is recalculated from (5).

\section{APPLICATION}

\section{A. Machine studied}

A 5-phase buried permanent magnet synchronous machine is considered. The machine has 7 pole pairs and 20 slots leading to a fractional number of slots per pole pair. The mesh of the ferromagnetic part is given in Fig. 1 and the number of elements is equal to $n_{e}=37552$.

\section{B. Evaluation of (D)EIM combined with Maxvol technique}

In order to evaluate the proposed method of sensor placement for the reconstruction of the magnetic flux distribution, we consider the operating point at no load. The supply currents of the machine are equal to zero, the magnetic field is only created by the permanent magnets. We consider a rotation interval of $360 / 7^{\circ}$, which corresponds to an electrical period. The field distribution $\mathbf{B}_{\mathrm{j}}(1 \leq \mathrm{j} \leq \mathrm{N})$ has been calculated for $\mathrm{N}=90$ angular positions uniformly distributed on this interval. We have then constructed the $2 \mathrm{n}_{\mathrm{e}} \times \mathrm{N}$ matrix of snapshots $\boldsymbol{B}$ by concatenating the $2 n_{e} \times 1$ vectors $\mathbf{B}_{j}(1 \leq j \leq N)$. Then, we have applied the (D)EIM and the Maxvol technique (see II-B and II-C) to determine the position of the M sensors $\varphi$ as well the reconstruction matrix $\boldsymbol{R}$ (see (2)). In order to evaluate the quality of the reconstructed field as a function of the number of sensors $\mathrm{M}$, we define the reconstruction error $\varepsilon_{M}$ :

$\varepsilon_{M}=\log \left(\sqrt{\frac{\sum_{\mathrm{j}=1}^{\mathrm{N}}\left\|\mathbf{B}_{\mathrm{j}}-\mathbf{B}_{\mathrm{j}}^{\text {reconst }, \mathrm{M}}\right\|^{2}}{\sum_{\mathrm{j}=1}^{\mathrm{N}}\left\|\mathbf{B}_{\mathrm{j}}\right\|^{2}}}\right)$

With $\mathbf{B}_{\mathrm{j}}^{\text {reconst, } \mathrm{M}}$ being the reconstruction field at the $\mathrm{j}^{\text {th }}$ position using $M$ sensors. We have calculated the error $\varepsilon_{M}$ when applying the (D)EIM alone and when combining the (D)EIM with the Maxvol technique. We present in Fig. 2 the evolution of the error $\varepsilon_{M}$ as a function of the sensor number $\mathrm{M}(1 \leq \mathrm{j} \leq \mathrm{N}$ 1). We do not consider the case $\mathrm{M}=\mathrm{N}$ because, by principle of the (D)EIM, the error $\varepsilon_{M}$ is equal to zero. We can see that the (D)EIM and (D)EIM/Maxvol method has almost the same behavior with a decrease of the error as a function of the sensor number which tends to zero. We can see also that the Maxvol technique enables to improve slightly the quality of the reconstruction by relocating more conveniently the sensors. It should be noticed that the (D)EIM is very fast, even combined with the Maxvol technique, leading to very short computation time of $\boldsymbol{\varphi}$ and $\boldsymbol{R}$ whatever the number of sensors (less than 1 minute with Scilab® for the proposed example).

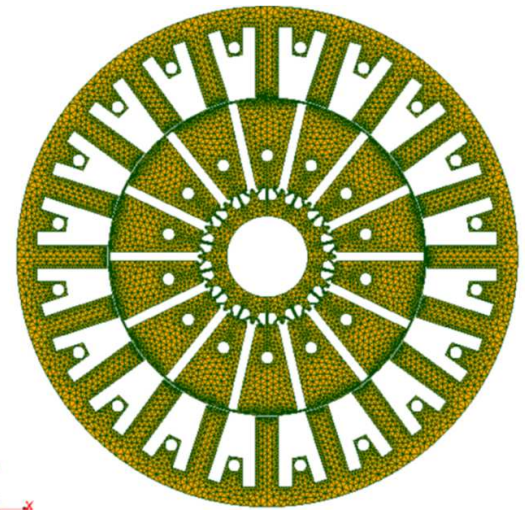

Fig.1. Geometry and mesh of the ferromagnetic parts of the studied machine

\section{Location of the sensors}

We also study the evolution of the position of the sensors as a function of the number of sensors. In Fig. 3, we indicate the 
location of the sensors for $M=20$. We can see that all of them are close to the airgap and that the number of sensors measuring either the radial $\left(M_{r}=11\right)$ or the tangential $\left(M_{s}=9\right)$ components of the magnetic flux density are almost the same. We present also the location, where the error $\varepsilon_{\max }$ which is equal to the maximum entry of the matrix $\boldsymbol{B}-\boldsymbol{B}^{\text {recons }}$, which corresponds to the next point that would be selected by the (D)EIM. We can see that it is still located in the airgap. Globally, whatever the number of sensors, we note that the sensors are almost always located in the airgap or very close to it either on the rotor or stator side. The field distribution varies not so much in the stator and in the rotor as a function of the position, whereas this variation is much more complex within the airgap or close to it leading to always a higher difference between the snapshot $\boldsymbol{B}$ and the reconstructed field $\boldsymbol{B}^{\text {recons }}$ in the airgap. This difference being the criterion for the choice of the sensor position by the (D)EIM/Maxvol, the sensors are always chosen in that region.

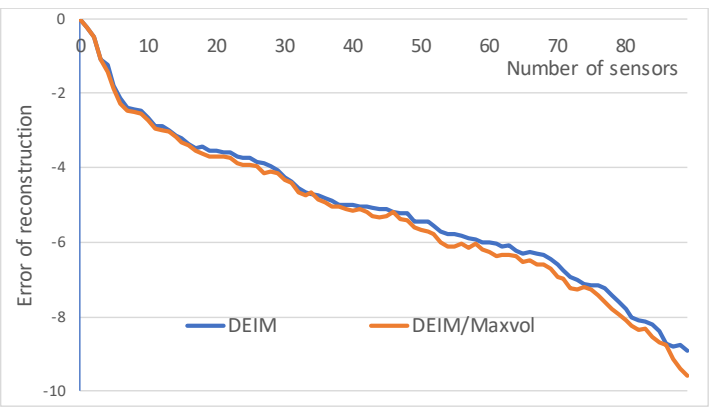

Fig.2. Evolution of the error $\varepsilon_{M}$ (see (6)) as a function of the number of sensors for the DEIM and DEIM/Maxvol

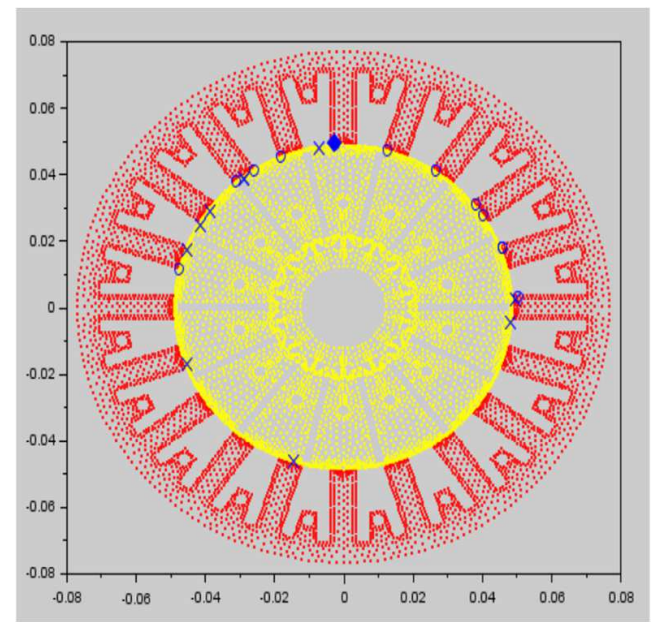

Fig.3. Locations of the sensors (o : radial component $\times$ : tangential component - : error maximum) for $\mathrm{M}=20$.

\section{Modification of the selection process}

For further practical applications of the proposed method, the sensors should be located in regions where it is easy to implement them. The airgap seems to be the best fitting region. The sensors can be inserted easily since the region is quite accessible. Moreover, this insertion can be done almost at the end of the process of fabrication before assembling the stator and the rotor together. It leads only to few modifications on the existing manufacturing processes to add the sensors.

It has been shown previously that the sensors are located in the airgap or very close to it without imposing any constraint on the position (The sensors should be located inside the machine). If we impose the sensors to be located inside the airgap, we can expect to keep a good quality of reconstruction. In order to evaluate it, we have run the (D)EIM/Maxvol technique by restricting the sensor location to the airgap. In Fig.4, we present the evolution of the error $\varepsilon_{M}$ (see (1)) as a function of the number of sensors when applying the (D)EIM/Maxvol technique with and without imposing the sensor location in the airgap. We can see an error decreasing according to $M$ in both cases. The comparison, with the original (D)EIM/Maxvol technique, shows that there is a small accuracy loss for a sensor number $\mathrm{M}$ lower than 20 but which remains acceptable (the maximum error ratio is equal to 1.2 for $M=7$ ). In the following, we will use only the (D)EIM/Maxvol/Airgap technique.

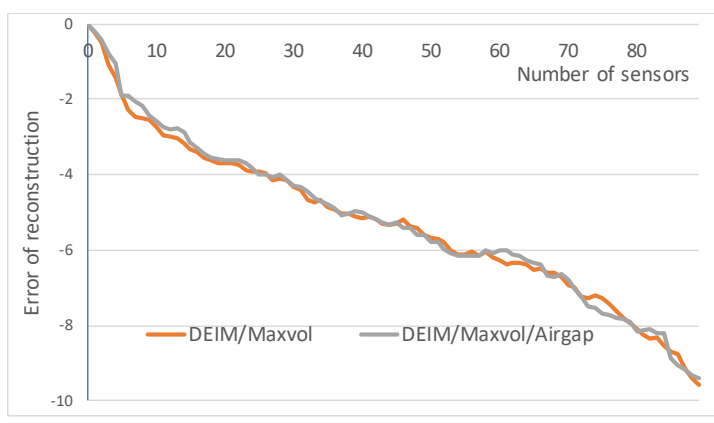

Fig.4. Comparison of the evolution of the error $\varepsilon_{M}$ (see (6)) as a function of the number of sensors obtained with the original DEIM/Maxvol technique and the DEIM/Maxvol/Airgap technique where the sensor locations are imposed in the airgap.

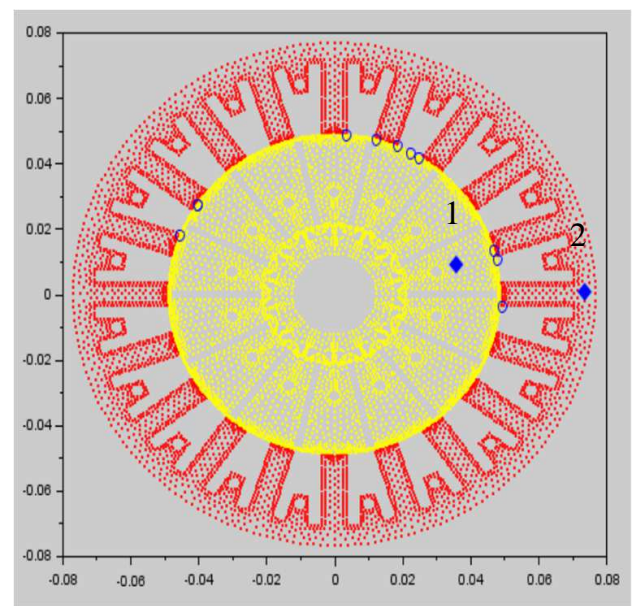

Fig.5. Locations of the sensors (o : radial component $\bullet$ : selected points to compare the evolution of the reconstructed field as a function of the number $\mathrm{M}$ ) for $\mathrm{M}=10$.

\section{E. Local field reconstruction}

We have reconstructed the magnetic flux density for different numbers $\mathrm{M}$ of sensors. In order to illustrate our method, we have selected to two points, presented in Fig.5, one located in the rotor and one in the stator. In Fig.6 and Fig.7, we have compared the evolution of the reconstructed components (radial 
and tangential) for three sensor numbers ( $M=5,10$ and 20) to the components obtained by the FE model, which is our reference. We can see that for $M=5$ the quality of the reconstructed field is quite poor. We can notice that a good approximation can be obtained with $M=10$ sensors in the stator (point 2). In Fig.5, we present the sensor location in the airgap. However, in the rotor, it appears spurious oscillations on the radial components which can be met when applying interpolation method like the (D)EIM. However, we can see that with $\mathrm{M}=20$ sensors, we have a good agreement with the reference. This result can be generalized to the whole machine.

\section{CONCLUSION}

In this paper we have tested a method to automatically determine the sensor locations in order to reconstruct the magnetic flux density distribution in a permanent magnet machine as a function of the position. It appears surprisingly that the method places the sensors mainly in the airgap of the machine which will facilitate the insertion of such sensors inside the machine. The chosen machine has a fractional number of slots per pole pair leading to a complex evolution of the magnetic flux density distribution as a function of position of the rotor. Thus, to obtain an accurate reconstruction of the field on the whole machine, the sensor number is quite high. However, we have also seen if the targeting domain of field reconstruction is reduced (for example the stator yoke), this number can be reduced. It appears through the obtained results that it is potentially possible to reconstruct the magnetic flux density distribution in the machine by means of a limited number of sensors.

\section{ACKNOWLEDGMENT}

This work has been carried out within the framework of CE2I project. CE2I is co-financed by European Union with the financial support of European Regional Development Fund (ERDF), French State and the French Region of Hauts-deFrance.

\section{REFERENCES}

[1] D. W. M. Veldman, R. H. B. Fey, H. J. Zwart, M. M. J. van de Wal, J. D. B. J. van den Boom and H. Nijmeijer, "Sensor and Actuator Placement for Proportional Feedback Control in Advection-Diffusion Equations," in IEEE Control Systems Letters, vol. 4, no. 1, pp. 193-198, Jan. 2020

[2] M. Barrault, N. C. Nguyen, Y. Maday, A. T. Patera. "An "empirical interpolation method: Application to efficient reduced-basis discretization of partial differential equations", C. R. Acad. Sci. Paris, vol. 339(9), 2004.

[3] S. Chaturantabut, D. C. Sorensen, "Nonlinear Model Reduction via Discrete Empirical Interpolation", SIAM J. Sci. Comput., vol. 32(5), pp.2737-2764, 2010.

[4] J.-P.Argaud, B.Bouriquet, F.de Caso, H.Gong, Y.Maday, O.Mula, "Sensor placement in nuclear reactors based on the generalized empirical interpolation method", Journal of Computational Physics, vol. 363, pp 354-370, 2018

[5] T. Henneron and S. Clénet, "Model-Order Reduction of Multiple-Input Non-Linear Systems Based on POD and DEI Methods," in IEEE Transactions on Magnetics, vol. 51, no. 3, pp. 1-4, March 2015

[6] L. Montier, A. Pierquin, T. Henneron and S. Clénet, "Structure Preserving Model Reduction of Low-Frequency Electromagnetic Problem Based on POD and DEIM," in IEEE Transactions on Magnetics, vol. 53, no. 6, pp. 1-4, June 2017, Art no. 7205204, doi: 10.1109/TMAG.2017.2663761.
[7] S. A. Goreinov, I. V. Oseledets, D. V. Savostyanov, E. E. Tyrtyshnikov and N. L. Zamarashkin, "How to find a good submatrix". In Matrix Methods: Theory, Algorithms And Applications, World Scientic, 2010.
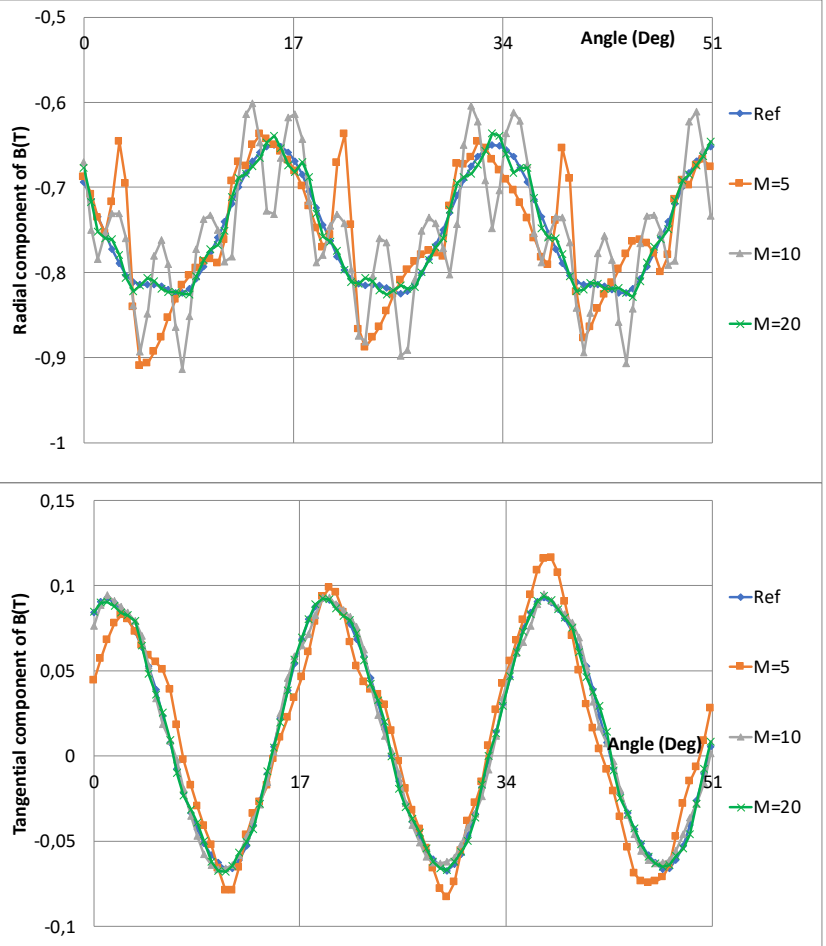

Fig.6. Radial (top) and tangential (bottom) components of the magnetic flux density reconstructed with different numbers of sensors $M$ compared to the reference computed with the FE model in the rotor (point 1 in Fig. 5).

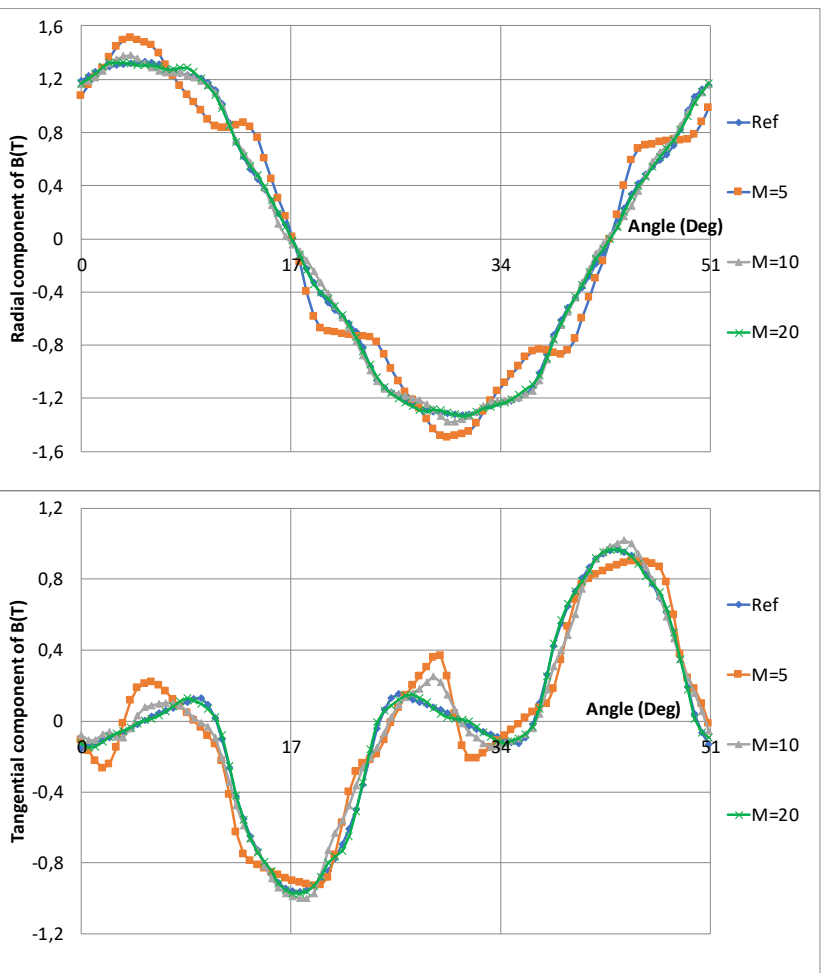

Fig.7. Radial (top) and tangential (bottom) components of the magnetic flux density reconstructed with different numbers of sensors $M$ compared to the reference computed with the FE model in the stator (point 2 in Fig. 5). 